\title{
Survey for Fe-Si in Apollo 16 Regolith Sample 61501,22
}

\author{
P. Gopon, J. Fournelle, M. Spicuzza, J.W. Valley \\ Dept. of Geoscience, University of Wisconsin, Madison, Wisconsin 53706 USA
}

Metallic iron (micron to submicron spherules) is relatively abundant in lunar regolith and is widely believed to have formed by reduction of Fe during space weathering (micrometeorite/ cosmic ray bombardment; [1]). Iron-silicides (Fe-Si) and native silicon require more reducing conditions than $\mathrm{Fe}^{0}$, and though predicted to be present in the lunar regolith have only been reported from one lunar meteorite and one lunar regolith sample [2,3,4]. Fe-Si and native silicon are only stable at extremely reducing conditions and have been proposed to form by a mechanism similar to that of metallic iron [3]. The stability of native silicon requires ten orders of magnitude lower oxygen fugacity than metallic iron at temperatures above $1600 \mathrm{~K}$ [5].

After the discovery of $\mathrm{Si}^{0}$ and Fe-Si in lunar regolith 61501,22 [2] and given the paucity of these phases in lunar samples reported in the literature, we systematically searched 61501,22 to locate additional FeSi specimens to determine their relative abundance and advance the understanding of their formation. Regolith grains for inspection were first selected under UV light (Apollo 16 regolith sample 61501,22) to re-create the conditions that led to the discovery of Fe-Si and $\mathrm{Si}^{\circ}$ by Spicuzza et al. [2]. Further study of lunar Fe-Si from Apollo samples, however has been limited to non-destructive methods because until now there was only one 60- $\mu$ m plagioclase grain containing $\mathrm{Fe}-\mathrm{Si}$ and $\mathrm{Si}^{\circ}$ from the original study [2].

During the initial analysis of Fe-Si [6], it was suggested that carbon ( 1-2 wt. \%, determined by EPMA) might be present in the Fe-Si, but the possibility of analytical artifacts has not been resolved. The presence of carbon was hypothesized to explain the ultra-reduced conditions. In order to determine with certainty the presence of carbon in these phases, a destructive technique (SIMS or atom probe) is necessary. Therefore we sought additional examples of Fe-Si/Si ${ }^{\circ}$ from 61501,22 to test this hypothesis,

We prepared 3 grain mounts of UV fluorescent grains (from 61501,22), in three size fractions (Mount 1: UV, 0.8-1mm, Mount 2: UV, 0.5-0.8mm, Mount 3: UV, $<0.5 \mathrm{~mm}$ ) as well as one mount with randomly selected UV and non-UV fluorescent grains (Mount 4: Random, $<0.5 \mathrm{~mm}$ ) to see if Fe-Si is limited to UV fluorescent grains. To date, two of the four mounts have been examined by SEM. In mount $3(<$ $0.5 \mathrm{~mm}$ UV fluorescent, figure 1, a-c), we found examples of Fe-Si (a few nm to $2 \mu \mathrm{m}$ in size, $\sim \mathrm{Fe}_{3} \mathrm{Si}$ ) in 10 grains (out of 441 grains). In mount 4 (Random $<0.5 \mathrm{~mm}$ ) we found a further 10 grains (out of 561 grains) containing Fe-Si. While we have not yet confirmed additional samples of $\mathrm{Si}^{0}$, these results increase by an order of magnitude the number of reported grains containing Fe-Si. Moreover, all found instances of Fe-Si were present encapsulated in a glassy matrix of anorthitic composition, referred to as glassy-anorthite (presumed glassy based on textural similarity to that present in [2]). Thus Fe-Si in this lunar regolith sample is relatively common, and may be more common in the lunar regolith in general than previously reported. 


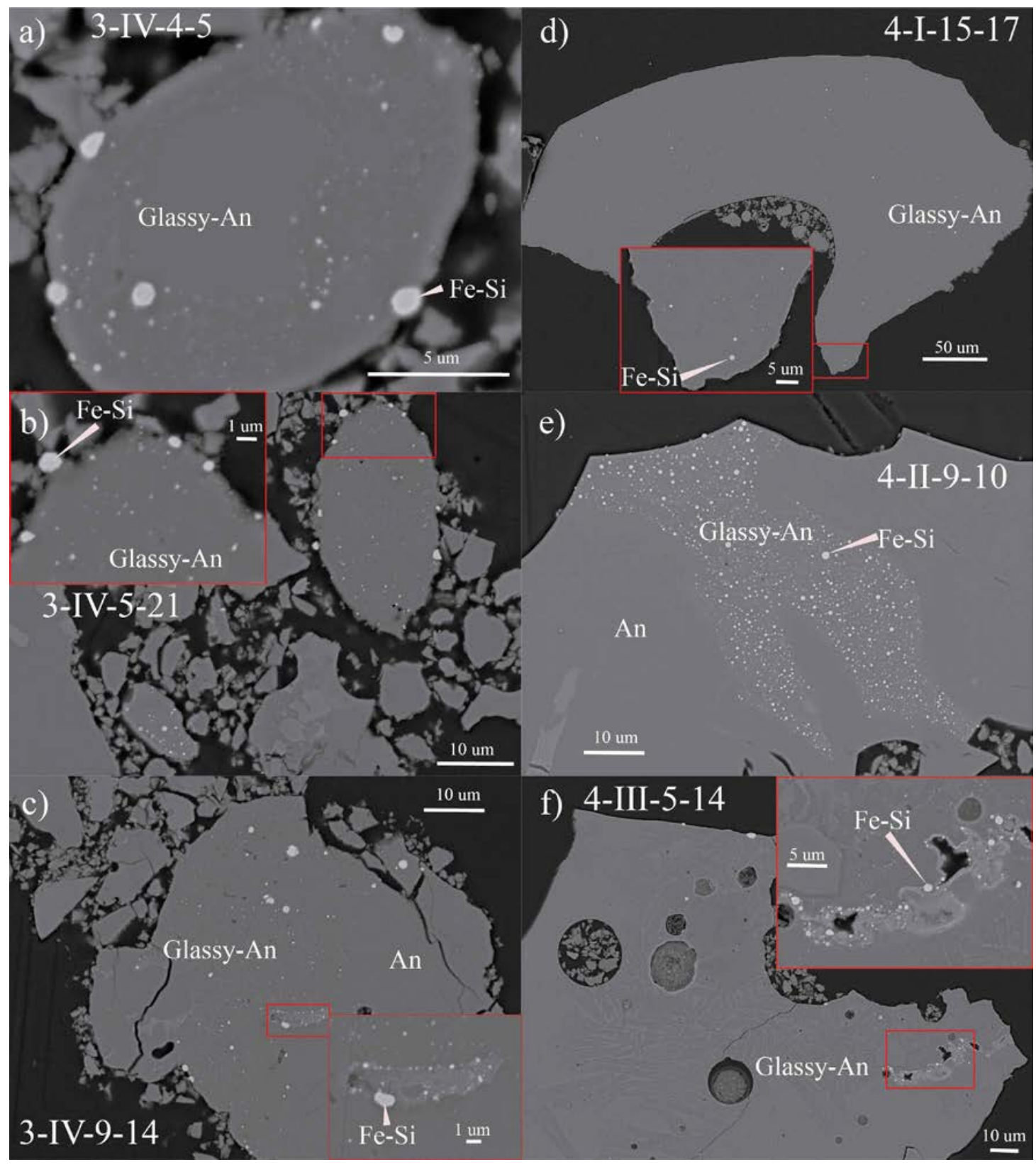

Figure 1: BSE images of Fe-Si occurrences in Apollo 16 sample 61501,22. Grain number is in the format of mount- quadrant-row-column. a) Glassy An. spherule containing Fe-Si. b) Two glassy An. grains in an agglutinate, the top grain contains Fe-Si blebs while the bottom only Fe-metal. c) glassy An. grain in agglutinate containing $\mathrm{Fe}-\mathrm{Si}$, the right half of the grain is crystalline anorthite. d) Glassy An. grain containing Fe-Si. e) Glassy An. grain containing a filled crack consisting of glassy-An. and Fe-Si blebs. f) Glassy An. grain containing Fe-S and Fe-mtl, Fe-Si is restricted to the highlighted vein feature.

References:

[1] Hapke, B., 2001, J. Geo. Research, v. 106, no. 10, 39-73.

[2] Spicuzza, M.J., et al., 2011, 42nd Lunar and Planetary Science Conference ( 2011 ), 16-17.

[3] Anand, M., et al., 2004, Proc. of the Nat. Acad. of Sci. USA, v. 101, no. 18, 6847-51.

[4] Nazarov et al., 2012, Petrology, v. 20, no. 20, 506-519

[5] Essene, E.J., and Fisher, D.C., 1986, Science (New York, N.Y.), v. 234, no. 4773, 189-93

[6] Gopon, P., et al., 2013, Microscopy and Microanalysis, 19(6), 1698-1708. 